\title{
AMBIÊNCIAS TÉRMICA, AÉREA E ACÚSTICA PARA REPRODUTORES SUÍNOS
}

\author{
YAMILIA B. TOLON ${ }^{1}$, MARTA S. BARACHO ${ }^{2}$, IRENILZA DE A. NÄÄS ${ }^{3}$, \\ MARCELO ROJAS ${ }^{4}$, DANIELLA J. DE MOURA ${ }^{5}$
}

\begin{abstract}
RESUMO: O ambiente de alojamento de instalações para reprodutores suínos tem influência direta no desempenho dos animais. Este trabalho teve como objetivo estimar um padrão de avaliação dos ambientes térmicos, aéreo e acústico em instalações para reprodutores de suínos (cachaços), de maneira que pudesse prover os animais de bem-estar. O experimento foi realizado em granja comercial localizada no município de Salto - SP. Foram levantados os dados de ambiências térmica, aérea e acústica do ambiente de criação, sendo analisados estatisticamente, tendo como base a condição de ambiência ideal para proporcionar bem-estar animal. Os resultados mostraram que a temperatura ambiente ficou cerca de $70 \%$ fora da normalidade, enquanto a umidade relativa do ar, a velocidade do ar e a concentração de gases estavam dentro da normalidade. Os dados de nível de ruídos, além de estarem dentro da faixa ideal, não apresentam variações muito altas. Com relação à análise feita utilizando lógica fuzzy, foi possível elaborar um cenário que indicou que os melhores índices de bem-estar para reprodutores suínos ocorrem quando o índice de conforto térmico se aproxima de $80 \%$, e quando o nível de ruído é menor do que $40 \mathrm{~dB}$. Em contrapartida, os piores índices de bem-estar ocorrem no setor em que os valores de conforto térmico são menores que $40 \%$, ao mesmo tempo em que o nível de ruído seja maior que $80 \mathrm{~dB}$, promovendo condições inadequadas ao animal e podendo interferir diretamente no desempenho do sistema reprodutivo.
\end{abstract}

PALAVRAS-CHAVE: machos reprodutores, conforto térmico, bem-estar de suínos.

\section{THERMAL, AERIAL, AND ACUSTIC ENVIRONMENT FOR BOAR HOUSING}

\begin{abstract}
The swine breeder rearing environment directly affects the animal's performance. This research had the objective of developing a thermal, aerial and acoustic environmental evaluation pattern for boar housing. The experiment was carried on a commercial swine farm in Salto County - SP, Brazil. Thermal, aerial and acoustic environment data of rearing conditions were registered. Data were statistically analyzed using as threshold the ideal housing environment that leads to animal welfare. Results showed that ambient temperature was around $70 \%$ beyond normal range, while air relative humidity, air speed and gases concentration were within threshold values. Noise level data besides being within normal range did not present large variation. In relation to the fuzzy logic analysis it was possible to build up a scenario which indicated that the best welfare indexes to male swine breeders happens when thermal comfort index are close to $80 \%$, and noise level is lower than $40 \mathrm{~dB}$. In the other hand the worst welfare index occur in the sector where the thermal comfort values are below $40 \%$ at the same time that the noise level is higher than $80 \mathrm{~dB}$ leading to inadequate conditions to the animal, and may directly interfere in the reproduction system performance.
\end{abstract}

KEYWORDS: swine male breeder, thermal comfort, breeder welfare.

\footnotetext{
${ }^{1}$ Zootecnista, Faculdade de Engenharia Agrícola, UNICAMP, Campinas - SP, Fone: (0XX19) 3521-1129, yamilia55@yahoo.com.br

${ }^{2}$ Bióloga, Pesquisadora Colaboradora, Faculdade de Engenharia Agrícola, UNICAMP, Campinas - SP.

${ }^{3}$ Eng $^{\mathrm{a}}$ Civil, Professora Titular Colaboradora, Faculdade de Engenharia Agrícola, UNICAMP, Campinas - SP.

${ }^{4}$ Aluno do Curso de Engenharia Agrícola, UNICAMP, Campinas - SP.

${ }^{5}$ Eng $^{\mathrm{a}}$ Agrícola, Professora Adjunta, Faculdade de Engenharia Agrícola, UNICAMP, Campinas - SP.

Recebido pelo Conselho Editorial em: 5-9-2008
}

Aprovado pelo Conselho Editorial em: 12-12-2009

Eng. Agríc., Jaboticabal, v.30, n.1, p.1-13,jan./fev. 2010 


\section{INTRODUÇÃO}

A temperatura ambiente é um dos importantes fatores ambientais que interfere na reprodução de suínos, e alterações no ambiente térmico podem levar à redução do desempenho e à incidência de patologia nos machos reprodutores (MIES FILHO, 1975; FOOTE, 1978; JAINUDEEN \& HAFEZ, 1995; HANSEN, 1999; HUANG et al., 2000; ROZEBOOM et al., 2000). A literatura internacional aponta que a umidade relativa também interfere na eficiência reprodutiva dos machos suínos e, quando associada a altas temperaturas, afeta a morfologia espermática, sendo um dos maiores problemas que atingem machos suínos sexualmente ativos, uma vez que o estresse induz a excessiva produção de corticoides (HENNESSY \& WILLIAMSON, 1983; WETTEMAN \& BAZER, 1985; SEREN et al., 1988; KUNAVONGKRIT \& PRATEEP, 1995; CIERESZKO et al., 2000; SURIYASOMBOON et al., 2006). CORCUERA et al. (2002) encontraram que o controle de fatores ambientais na instalação leva à redução das perdas de qualidade e de quantidade espermática, resultando em maior motilidade, enquanto a percentagem de acrossomas normais foram maiores em machos alojados em ambiente controlado.

Os gases gerados dentro do abrigo aos reprodutores também podem afetar o desempenho desses animais (ROBERTSON, 1990; HARDOIM, 1995; JENSEN, 2002). Dentre aqueles, os mais importantes relatados na literatura são a amônia $\left(\mathrm{NH}_{3}\right)$, o gás carbônico $\left(\mathrm{CO}_{2}\right)$ e o gás sulfídrico $\left(\mathrm{H}_{2} \mathrm{~S}\right)$ (BRUCE, 1981; PERDOMO et al., 2001; KRISTENSEN \& WATHES, 2001).

Suínos vocalizam em várias situações, e sua expressão vocal é citada como padrão de reconhecimento de estado de bem-estar, frustração ou sofrimento (MARTRENCHAR et al., 2000; MARX et al., 2003; SAMPAIO et al., 2005; SAMPAIO et al., 2007). Grunhidos (roncos) curtos e longos são a forma mais comum de vocalização de suínos, e essa classificação está relacionada ao enfrentamento de determinada situação que gere atenção, ou ainda exprima desconforto (MARCHANT et al., 2001; KASANEN \& ALGERS, 2002). A vocalização mostra potencial de ser utilizada para aferir as condições a que o animal está exposto (MOURA et al., 2008).

A lógica fuzzy é usada quando descrição do problema não pode ser feita de forma precisa. Essa metodologia tem sido aplicada em análise de dados, sistemas especialistas, além de uso em controle e em otimização (WEBER \& KLEIN, 2003). O uso da teoria dos conjuntos fuzzy tem sido promissor na área de engenharia (KLIR \& YUAN, 1995), e algumas aplicações foram tidas com êxito na área de ambiência animal, utilizando conceitos para simular e modelar informações (AMENDOLA et al., 2004; AMENDOLA et al., 2005; OLIVEIRA et al., 2005; OWADA et al., 2007).

Esta pesquisa teve como objetivo identificar os padrões ideais de ambiente de alojamento para machos suínos reprodutores que representem boas condições de bem-estar animal.

\section{MATERIAL E MÉTODOS}

O experimento foi conduzido em uma granja comercial de produção de suínos, situada a $23^{\circ} 12^{\prime} 10^{\prime \prime}$ de latitude sul, $47^{\circ} 17^{\prime} 35^{\prime \prime}$ de longitude oeste e altitude média de $521 \mathrm{~m}$, no período de maio a julho de 2007. Na Figura 1, mostra-se o interior da instalação estudada, que tinha telhado com telha de cerâmica, paredes construídas com blocos de cimento, altura do pé-direito de 3,00 m, e piso compacto com revestimento de argamassa. O número de baias era igual a dez, e a área total do galpão era de $135 \mathrm{~m}^{2}$ (15 m de comprimento por $9 \mathrm{~m}$ de largura). $\mathrm{O}$ macho reprodutor somente deixa a baia para coleta de sêmen, quando esse é direcionado, pelos funcionários, para sala onde está localizado o manequim. A inseminação é feita nas porcas em estro, utilizando pipeta do tipo "Melrose". 


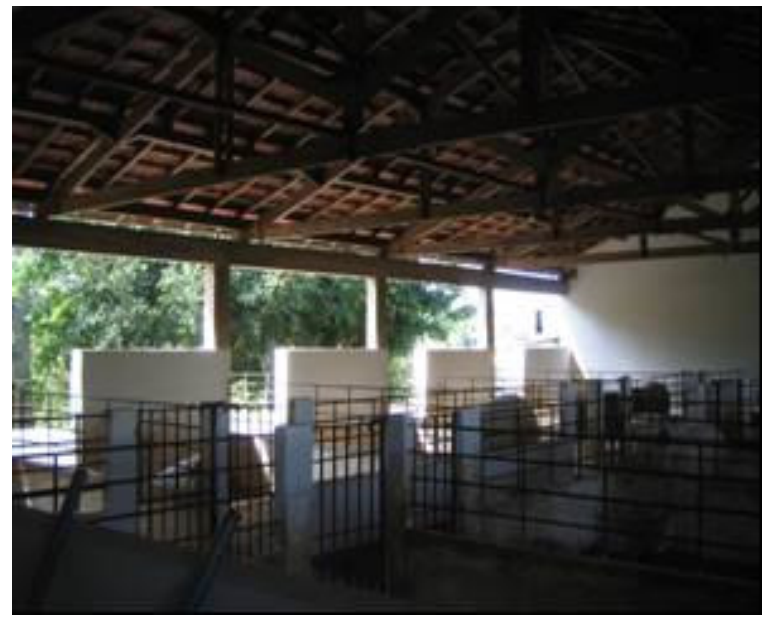

FIGURA 1. Vista interna da instalação de cachaço estudada. Internal view of the studied boar housing.

Foram coletadas, dentro da instalação para machos reprodutores, as seguintes variáveis: temperatura de bulbo seco (TBS), umidade relativa do ar (UR), velocidade do ar (VA), gás amônia $\left(\mathrm{NH}_{3}\right)$ e pressão sonora (RU). Os dados de TBS e UR foram registrados no centro geométrico da instalação, à altura de $1,5 \mathrm{~m}$ do piso, utilizando o monitor de estresse térmico da marca Questemp ${ }^{\circledR}$ 34. A velocidade do ar foi medida por meio de anemômetro, modelo HTA 4200, Marca Pacer ${ }^{\circledR}$. O nível de pressão sonora foi medido utilizando-se de decibelímetro Quest ${ }^{\circledR} 2900$.

A análise dos dados coletados foi feita com o auxílio do software MINITAB ${ }^{\circledR}$ Release 14 (2004). Essa análise foi baseada em dados de condições ambientais ótimas para o interior de edificações de cachaços (Tabela 1), utilizando informação da literatura corrente, após o resultado da análise estatística de adequação/conformidade.

TABELA 1. Condições ambientais ideais de alojamento de machos suínos. Ideal environmental housing conditions for swine male breeders.

\begin{tabular}{cc}
\hline Dados do Ambiente & Valores Recomendados como Ideais \\
\hline TBS $\left({ }^{\circ} \mathrm{C}\right)$ & $10-16$ \\
UR $(\%)$ & $60-70$ \\
$\mathrm{NH}_{3}(\mathrm{ppm})$ & $0-10$ \\
VA $\left(\mathrm{m} \mathrm{s}^{-1}\right)$ & $0,2-0,4$ \\
RU $($ slow, dB) & $0-85$ \\
\hline
\end{tabular}

Fonte: BENEDI (1996), CIGR (1994) e SAMPAIO (2004)

Utilizou-se da Teoria dos Conjuntos Fuzzy para, interconectando as diferentes variáveis, obter como variável-resposta o bem-estar dos machos. Inicialmente, buscou-se obter como variável-resposta o conforto térmico (CT), a partir das variáveis TBS e UR. O nível de pressão sonora, sob a forma de ruído emitido, foi usado como variável dependente. Tanto UR como TBS e RU foram transformadas em Funções de Pertinência Fuzzy a partir do conhecimento de especialistas, além de dados disponíveis na literatura (BENEDI, 1996; CIGR, 1994; SAMPAIO, 2004).

Foram construídas bases de regras com pesos, em função de sua coesão com os dados. O método usado para a geração das funções de pertinência foi o de Mandani, e a defuzzificação foi feita pelo método do centro de gravidade.

\section{RESULTADOS E DISCUSSÃO}

Os resultados da análise de adequação dos valores registrados, especificando os valores mínimos e máximos ideais para as edificações de cachaços, encontram-se na Figura 2. No gráfico, 
as faixas em vermelho indicam o limite inferior de especificação (LSL), o limite superior de especificação (USL) e o valor ideal (VI). A curva resultante mostra que os valores de temperatura ambiente coletados estão deslocados um pouco acima do ideal $\left(10{ }^{\circ} \mathrm{C}-16^{\circ} \mathrm{C}\right)$, tendo como valor médio de temperatura $19,6^{\circ} \mathrm{C}$.

O gráfico mostra, também, que os valores estão dispersos, com alta amplitude entre o menor e o maior valor. Dos dados coletados, $70 \%$ estão fora do intervalo ideal de temperatura (target); sendo assim, quando comparados com os valores ideais, podem ser classificados como inadequados. Tanto para inseminação artificial como para monta natural, a temperatura ambiente ideal para os cachaços está entre 18 e $20^{\circ} \mathrm{C}$, e umidade relativa, de 60 a $70 \%$. Temperaturas superiores a $25^{\circ} \mathrm{C}$ e altas variações (superiores a $6{ }^{\circ} \mathrm{C}$ ) podem comprometer a qualidade do sêmen, criando problemas de baixa fertilidade e prolificidade. Essa discrepância da temperatura ambiente encontrada neste trabalho pode ter influência negativa sobre a qualidade e a quantidade do sêmen, pois, de acordo com vários autores, tal situação pode aumentar a percentagem de espermatozoides com defeitos morfológicos e ainda reduzir a produção de espermatozoide (MCNITT et al., 1972; LARSSON \& EINARSSON, 1984; MALMGREN, 1988).

JAINUDEEN \& HAFEZ (1995) observaram que temperaturas acima da faixa ideal ocasionam menor volume total do ejaculado, produção reduzida de espermatozoides e maior ocorrência de espermatozoides com gota plasmática proximial. Também há indicações de que a variação de temperatura durante o dia, que é comum principalmente nos estados do sul do País, durante determinados períodos do ano, constitui importante fator de estresse para os animais (SCHNEIDER, 2007).

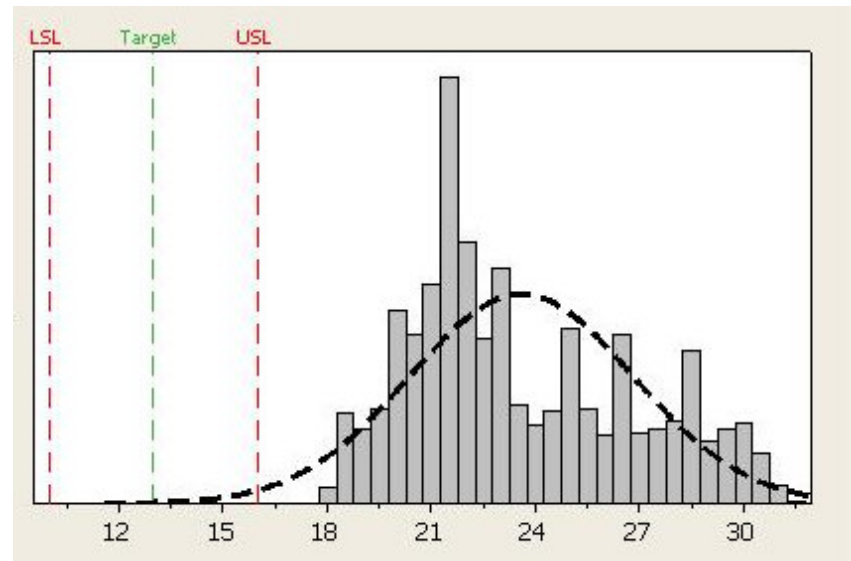

FIGURA 2. Gráfico de adequação dos dados de temperatura de bulbo seco. Curve fitting for dry bulb temperature data.

Com relação aos valores de UR, a curva (Figura 3) mostra que os dados de umidade relativa coletados estão deslocados um pouco acima do ideal (60\%-70\%), tendo como valor médio 74,07\%.

Os valores encontrados estão dispersos, com amplitude muito elevada entre o menor e o maior valor. Dos dados coletados, $21,21 \%$ estão abaixo do intervalo ideal de umidade relativa, e $65,99 \%$ acima, sendo que $78,05 \%$ de dados estão fora da faixa ideal. Sendo assim, quando comparados com os valores ideais de UR, esses podem ser classificados como inadequados por estarem fora da faixa ideal (target) e apresentarem dispersão alta. Com isso, determinou-se que a UR esteve predominantemente acima da faixa ideal. SURIYASOMBOON et al. (2005) e SURIYASOMBOON et al. (2006) observaram que elevadas umidades relativas ambientais, associadas com temperaturas elevadas, ocasionam redução no número de espermatozoides normais e aumento no número de espermatozoides com gota plasmática proximial e distal, prejudicando o desempenho dos reprodutores. 


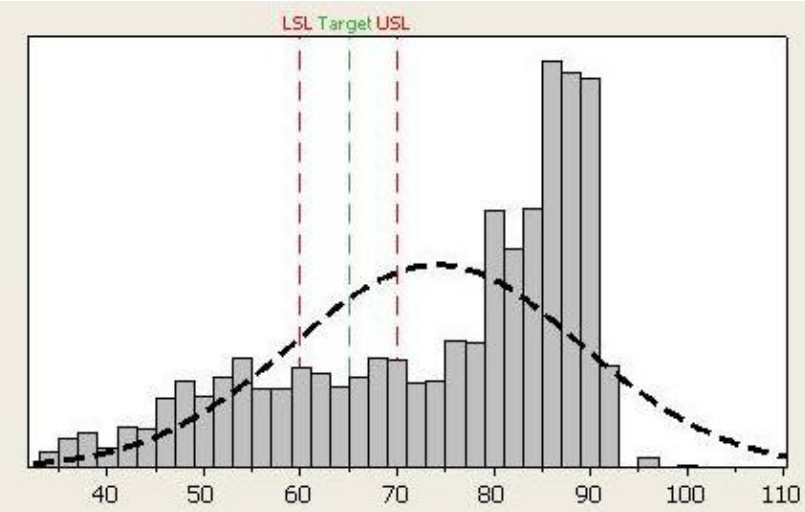

FIGURA 3. Gráfico de adequação dos dados de umidade relativa. Curve fitting for relative humidity data.

Os valores registrados de VA estão dentro da faixa do ideal $\left(0,2 \mathrm{~m} \mathrm{~s}^{-1}-0,4 \mathrm{~m} \mathrm{~s}^{-1}\right)$, tendo como valor médio $0,38 \mathrm{~m} \mathrm{~s}^{-1}$ (Figura 4). Os valores obtidos indicam dispersão alta e amplitude elevada entre o menor e o maior valor. Dos dados coletados, 37,5\% estão abaixo do intervalo ideal (target) e $41,67 \%$ acima, sendo que $80,80 \%$ de dados estão fora da faixa ideal de velocidade do ar. Valores muito altos de velocidade do ar no ambiente de alojamento podem ocasionar estresse nos animais, e a ausência total de movimentação de ar pode dificultar a troca de calor e a dispersão de gases da instalação.

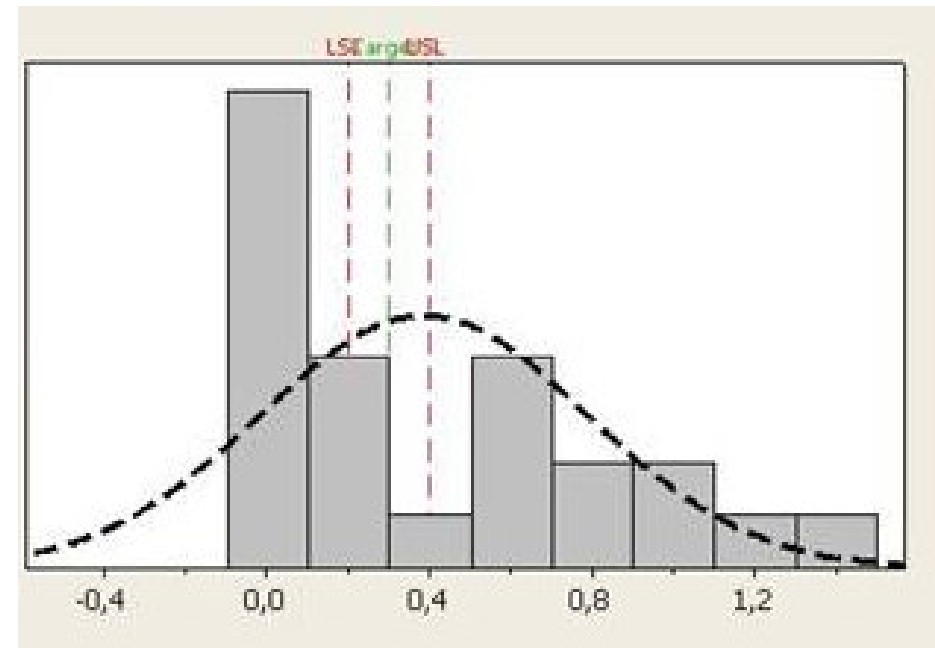

FIGURA 4. Gráfico da adequação dos dados de velocidade do ar. Curve fitting for air speed data.

Com relação aos resultados da concentração de $\mathrm{NH}_{3}$ na instalação, na Figura 5, mostra-se que os valores registrados estão dentro da faixa ideal (0-10), tendo como valor médio $2 \mathrm{ppm}$. Os dados, além de estarem dentro da faixa ideal, também não apresentam variação alta e, tanto os dados coletados quanto a estimativa (target), estão $100 \%$ dentro da faixa de concentração ideal para a amônia. Com isso, pode-se dizer que os valores de concentração de amônia medidos na instalação dos cachaços estão abaixo dos níveis máximos admissíveis, sendo adequados ao bem-estar animal, conforme preconiza JENSEN (2002), que relaciona o ganho de pesos em animais à redução desse gás no ambiente em que os mesmos estão alojados. 


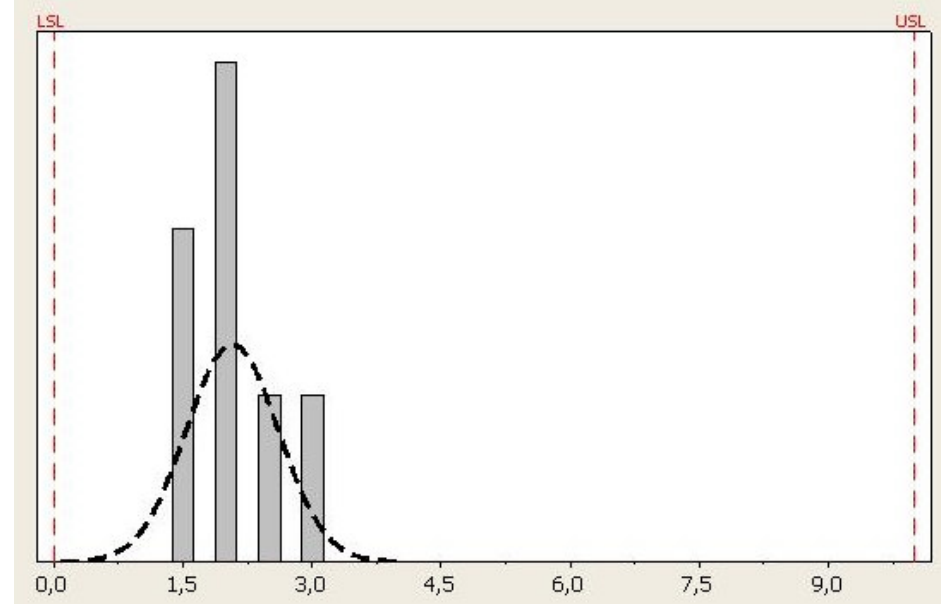

FIGURA 5. Gráfico de análise da concentração de amônia. Analysis graph for ammonia concentration data.

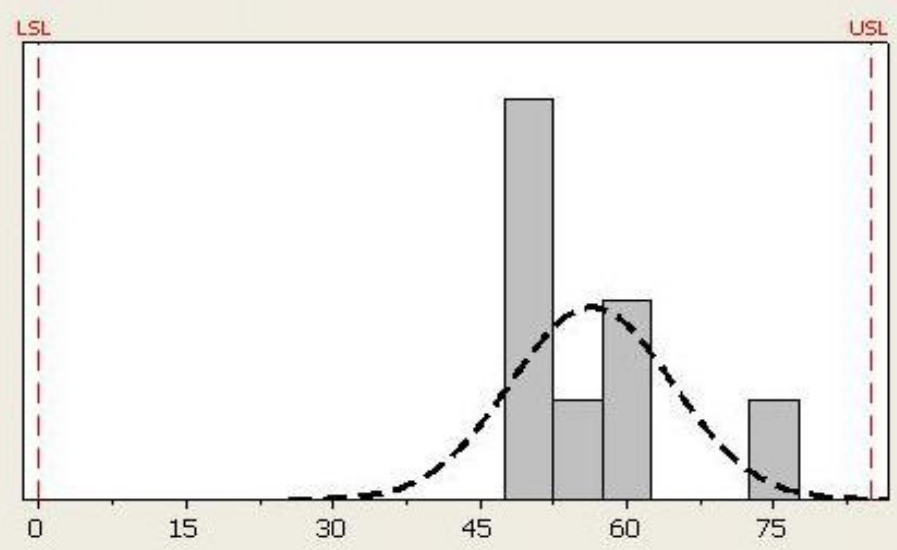

FIGURA 6. Gráfico de análise de nível de ruído. Analysis graph for noise level data.

Foram apenas medidos os níveis de ruídos (modo slow) que representam os ruídos contínuos, que causam maiores danos à saúde humana. Utilizando os dados de ruídos ideais, mostrados na Tabela 1, foi possível determinar a faixa de valores ótima e comparar com a curva dos dados (Figura 6). A curva mostra que os valores de ruídos estão dentro da faixa ideal (0-85), tendo como valor médio 56,38 dB. O gráfico da Figura 6 mostra, também, que os dados, além de estarem dentro da faixa ideal, não apresentam variações muito altas. Com isso, pode-se dizer que os níveis de ruídos na instalação de cachaços, abaixo de $85 \mathrm{~dB}$, não oferecem risco para a saúde humana e ao bem-estar animal. Deve-se ressaltar que, como não existem normas específicas para avaliar o limite de tolerância a ruídos pelos animais, têm sido adotados como valor ideal os mesmos níveis de ruídos recomendados para o ser humano.

Com os dados encontrados neste trabalho e os estabelecidos pela literatura, utilizou-se da Teoria dos Conjuntos Fuzzy para construir um modelo, segundo o qual um conjunto de valores expressos em uma dada escala é convertido em outro comparável, expresso em escala normalizada (ZADEH, 1965; EASTMANN, 1997; PANDORFI et al., 2005; OLIVEIRA et al., 2005). Neste caso específico, descreveu-se o modelo de ambiência ideal para alojamento de reprodutores suínos.

As regras para a construção das funções de pertinência fuzzy foi organizada com os resultados anteriores, além do auxílio de um especialista e da utilização de dados disponíveis na literatura (Tabela 2). 
TABELA 2. Faixas das variáveis de entrada e de saída de conforto térmico para reprodutores suínos. Variables zones of input and output of thermal comfort for breeders.

\begin{tabular}{cccccc}
\hline TBS $\left({ }^{\circ} \mathrm{C}\right)$ & Denominação & UR $(\%)$ & Denominação & Índice de CT $(\%)$ & Denominação \\
\hline abaixo de 8 & Muito Baixa & abaixo de 50 & Muito Baixa & abaixo de 20 & Muito Ruim \\
entre 8 e 10 & Baixa & entre 50 e 60 & Baixa & entre 20 e 40 & Ruim \\
entre 10 e 12 & Média & entre 60 e 65 & Média & entre 40 e 60 & Médio \\
entre 12 e 15 & Alta & entre 65 e 70 & Alta & entre 60 e 80 & Bom \\
acima de 15 & Muito Alta & acima de 70 & Muito Alta & acima de 80 & Muito Bom \\
\hline
\end{tabular}

A Tabela 3 foi gerada pelas variáveis de entrada TBS e UR, tendo como variável de saída o índice de conforto térmico (CT). Os valores de CT foram acompanhados pelos pesos da regra citada, em que 1 representa 100\% de exatidão. Na Figura 7, ilustra-se a combinação das 25 regras utilizadas para a estimativa de CT, enquanto, na Figura 8, mostra-se a superfície fuzzy de CT, em função de valores de TBS e UR.

TABELA 3. Faixas de índice de conforto térmico como variável resposta de TBS e UR, em alojamento de reprodutores suínos. Thermal comfort index zones as output variable of dry bulb temperature (DBT) and relative humidity (RH).

\begin{tabular}{cccccc}
\hline UR ITBS & TBS Muito Baixa & TBS Baixa & TBS Média & TBS Alta & TBS Muito Alta \\
\hline UR Muito Baixa & Muito Ruim $(0,8)$ & Ruim $(0,7)$ & Médio $(0,9)$ & Ruim $(0,7)$ & Muito Ruim $(0,7)$ \\
\hline UR Baixa & Ruim $(0,7)$ & $\begin{array}{c}\text { Médio }(0,9) \\
\text { Ruim }(0,2)\end{array}$ & Bom $(0,9)$ & $\begin{array}{c}\text { Médio }(0,9) \\
\text { Ruim }(0,1)\end{array}$ & Ruim $(0,7)$ \\
\hline UR Média & Médio $(0,7)$ & Rico $(0,7)$ & Muito Bom $(1,0)$ & Bom $(0,8)$ & Médio $(0,8)$ \\
\hline UR Alta & Ruim $(0,7)$ & $\begin{array}{c}\text { Médio }(0,9) \\
\text { Ruim }(0,3)\end{array}$ & Rico $(0,6)$ & $\begin{array}{c}\text { Médio }(0,9) \\
\text { Ruim }(0,4)\end{array}$ & Ruim $(0,7)$ \\
\hline UR Muito Alta & Muito Ruim $(0,9)$ & Ruim $(0,7)$ & Médio $(0,6)$ & Ruim $(0,7)$ & Muito Ruim $(1,0)$ \\
\hline
\end{tabular}

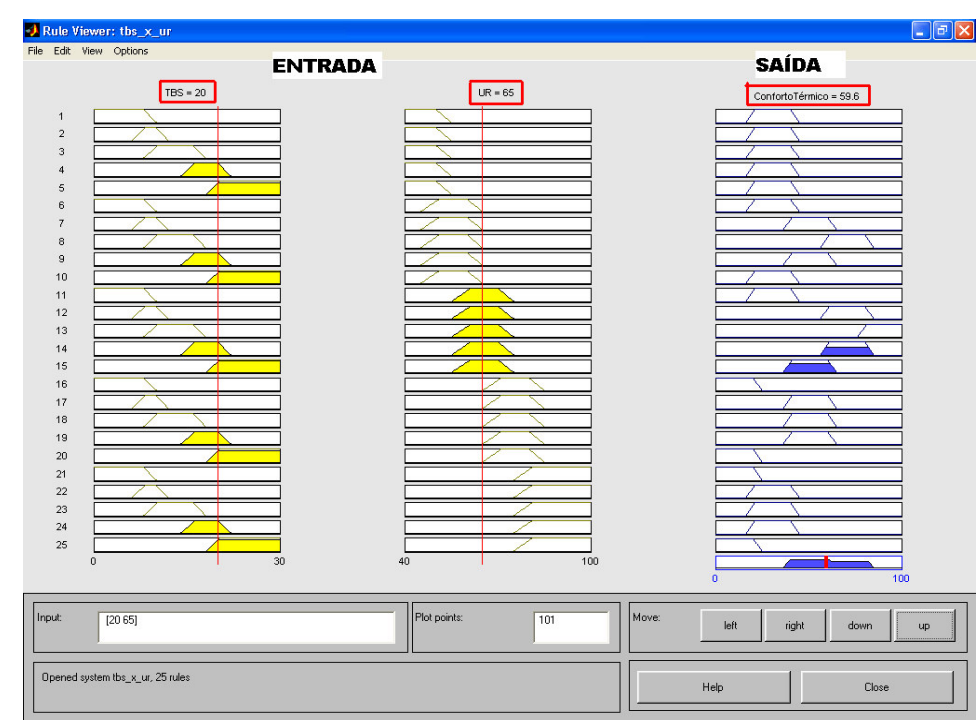

FIGURA 7. Resultados de defuzzificação para a determinação de CT para reprodutores suínos alojados, usando o método do centro de gravidade. Results of defuzzification for determining reared swine breeder thermal comfort (TC) using the gravity center method. 


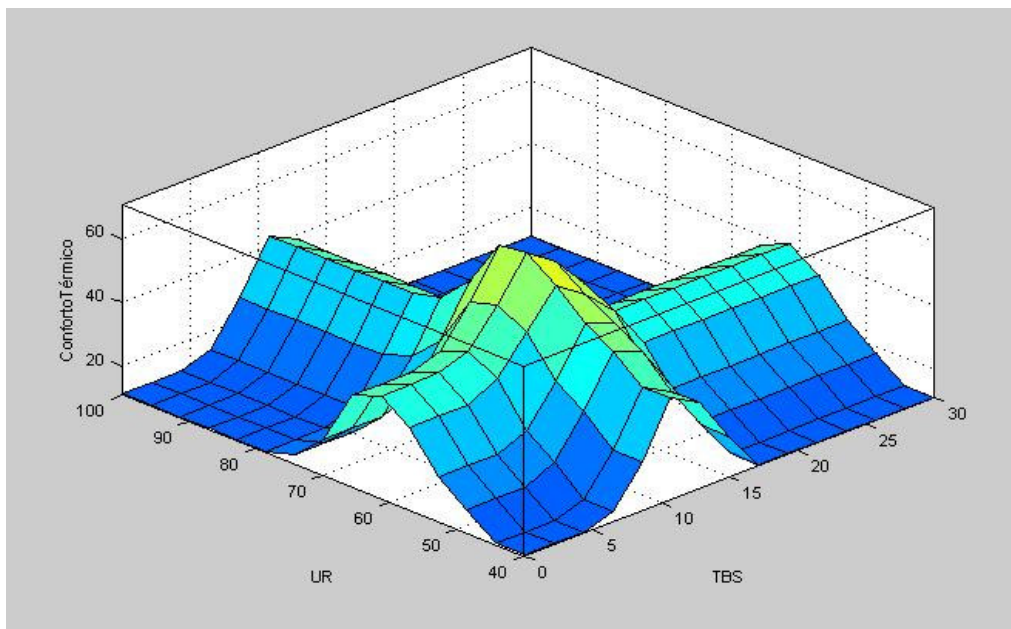

FIGURA 8. Superfície fuzzy de conforto térmico em alojamento de reprodutores suínos, gerada em função de TBS e UR. Fuzzy surface for thermal comfort of reared swine breeders generated as function of dry bulb temperature (DBT) and relative humidity (RH).

A superfície fuzzy da Figura 8 mostra a variação de CT em função de TBS e UR. Os valores de TBS vão de $0{ }^{\circ} \mathrm{C}$ a $30{ }^{\circ} \mathrm{C}$, enquanto os valores de UR variam de $40 \%$ a $100 \%$, de forma que, quanto mais baixos os valores na superfície, pior o índice de CT, e quanto mais acima na superfície, melhor o valor de CT. Os resultados da análise do conforto térmico (CT), tendo como entrada o nível de ruídos (RU), leva a uma variável de saída, que foi chamada de BEM-ESTAR. Na Tabela 4, mostram-se as faixas e as denominações de CT, RU e BEM-ESTAR, tendo sido gerada a Tabela 5 pelas variáveis de entrada CT e RU, tendo como variável de saída BEM-ESTAR.

TABELA 4. Denominação das faixas das variáveis de entrada (RU) e de saída de BEM-ESTAR, para alojamento de reprodutores de suínos. Denomination of the input variables of noise level (NL) and the output WELFARE for reared swine breeders.

\begin{tabular}{cccccc}
\hline CT (\%) & Denominação & RU (dB) & Denominação & Bem-Estar (\%) & Denominação \\
\hline abaixo de 20 & Muito Ruim & abaixo de 30 & Muito Baixo & abaixo de 20 & Muito Ruim \\
\hline entre 20 e 40 & Ruim & entre 30 e 65 & Baixo & entre 20 e 40 & Ruim \\
entre 40 e 60 & Médio & entre 65 e 80 & Média & entre 40 e 60 & Médio \\
\hline entre 60 e 80 & Bom & entre 80 e 90 & Alto & entre 60 e 80 & Bom \\
acima de 80 & Muito Bom & acima de 90 & Muito Alto & acima de 80 & Muito Bom \\
\hline
\end{tabular}

TABELA 5. Base de regras de determinação de BEM-ESTAR em função do nível de ruídos em alojamento de reprodutores de suíno. Base of rules for determining WELFARE as function of noise level in swine breeder rearing.

\begin{tabular}{|c|c|c|c|c|c|}
\hline $\mathrm{RU} \backslash \mathrm{CT}$ & CT Muito Baixo & CT Baixo & CT Média & CT Alto & CT Muito Alto \\
\hline Ruído Muito Baixo & $\begin{array}{l}\text { Médio }(0,6) \\
\text { Ruim }(0,5)\end{array}$ & Médio $(0,7)$ & $\operatorname{Bom}(0,8)$ & Muito $\operatorname{Bom}(0,7)$ & Muito Bom $(1,0)$ \\
\hline Ruído Baixo & $\begin{array}{l}\text { Ruim }(0,7) \\
\text { Médio }(0,4)\end{array}$ & Médio $(0,8)$ & $\begin{array}{c}\text { Bom }(0,7) \\
\text { Médio }(0,5)\end{array}$ & Bom $(0,9)$ & Muito Bom $(0,7)$ \\
\hline Ruído Médio & Ruim $(0,8)$ & $\begin{array}{l}\text { Ruim }(0,7) \\
\text { Médio }(0,5)\end{array}$ & Médio $(1,0)$ & $\begin{array}{c}\text { Bom }(0,7) \\
\text { Médio }(0,5)\end{array}$ & Bom $(0,8)$ \\
\hline Ruído Alto & Muito Ruim $(0,7)$ & Ruim $(0,9)$ & $\begin{array}{c}\text { Ruim }(0,7) \\
\text { Médio }(0,5)\end{array}$ & Médio $(0,8)$ & Médio $(0,7)$ \\
\hline Ruído Muito Alto & Muito Ruim $(1,0)$ & Muito Ruim $(0,7)$ & Ruim $(0,8)$ & $\begin{array}{l}\text { Ruim }(0,7) \\
\text { Médio }(0,4)\end{array}$ & $\begin{array}{c}\text { Médio }(0,6) \\
\text { Ruim }(0,5)\end{array}$ \\
\hline
\end{tabular}


Na Figura 9, mostra-se a defuzzificação pelo método de gravidade, enquanto na Figura 10, ilustra-se a superfície fuzzy gerada.

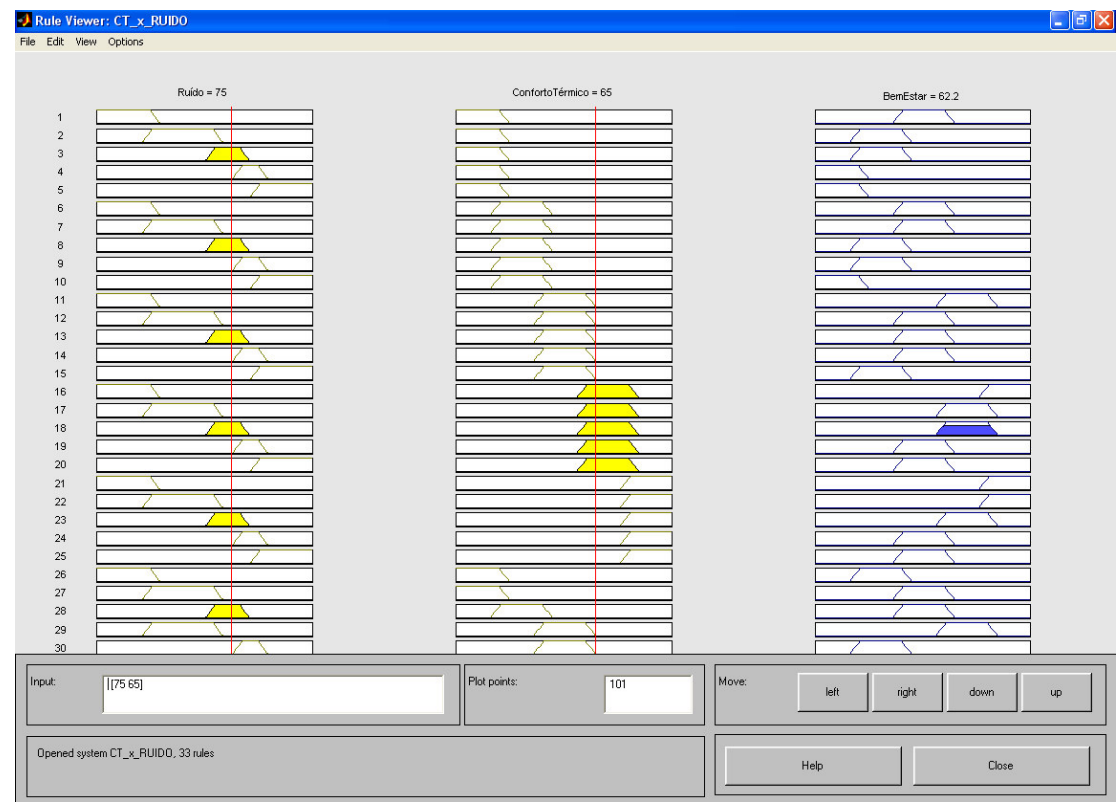

FIGURA 9. Resultado da defuzzificação usando o método de centro de gravidade para a saída BEM-ESTAR. Result of defuzzyfication using the gravity center method for the output WELFARE.

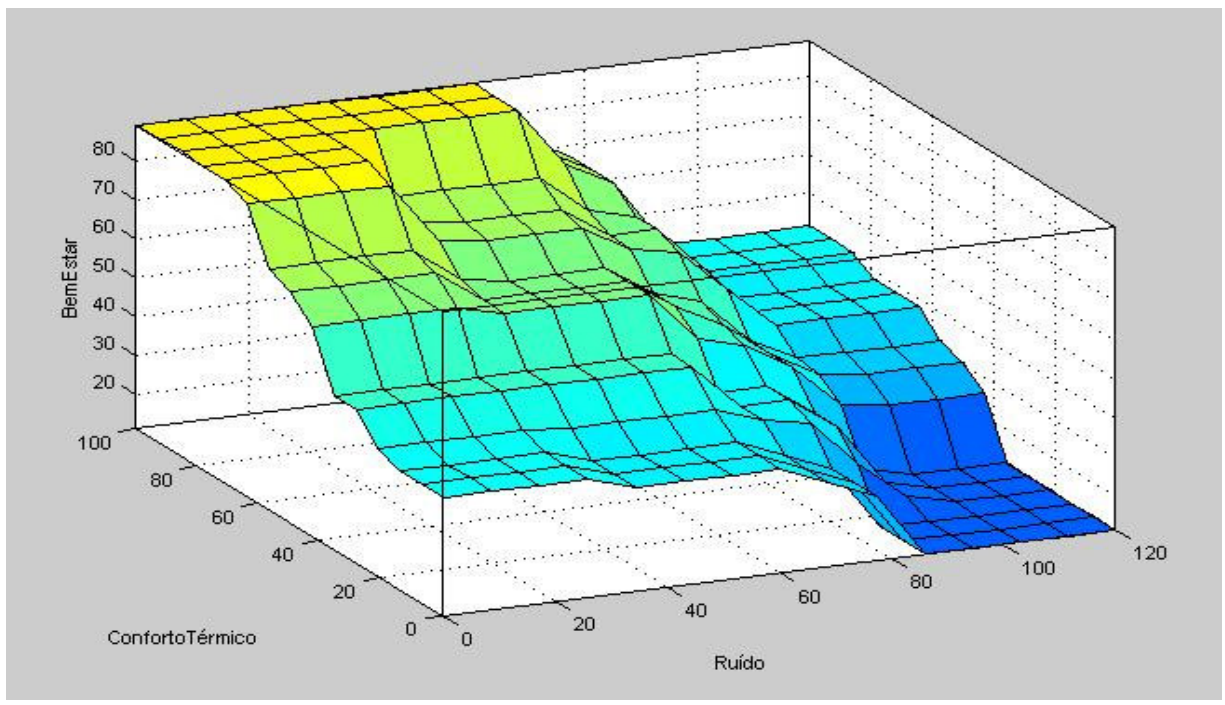

FIGURA 10. Superfície fuzzy de BEM-ESTAR para alojamento de reprodutores suínos, gerada em função de CT e RU. WELFARE fuzzy surface for swine breeder rearing generated as function of TC and NL.

Nota-se, pela superfície da Figura 10, que os melhores índices de bem-estar para reprodutores suínos ocorrem quando o conforto térmico CT se aproxima de $80 \%$, ao mesmo tempo em que os níveis de RU sejam menores do que $40 \mathrm{~dB}$. Em contrapartida, os piores índices de bem-estar ocorrem no setor em que os valores de CT são menores que $40 \%$, ao mesmo tempo em que os níveis de ruído sejam maiores que $80 \mathrm{~dB}$, promovendo condições inadequadas ao animal e podendo interferir negativamente no sistema reprodutivo. Embora os dados de ruído estejam nos limites recomendados pelas normas para humanos, NR-15 (1978), deve-se ressaltar que o ruído pode ser considerado indicador de bem-estar, devendo-se evitar, nos locais que os suínos são mantidos, 
níveis de ruído contínuo maior ou igual a $85 \mathrm{~dB}$. Também devem ser evitados ruídos constantes ou súbitos (RELATÓRIO, 2001), pois o animal, na presença desses níveis de pressão sonora, pode ficar impossibilitado de desenvolver seu sistema natural de comportamento.

O ambiente acústico, em confinamento de suínos, está relacionado ao ruído produzido principalmente pelos próprios animais, como resposta a algum agente agressivo, conforme apontam MANTEUFFEL \& SCHÖN (2002) e NÄÄS et al. (2008).

Em consequência à situação de desconforto, ele redirecionará sua postura para atitudes anômalas (MACHADO FILHO, 1988) e alterações fisiológicas, que estão correlacionadas. O comportamento alterado leva à diminuição da saúde do animal e, portanto, à propagação de doenças e pode ser indicativo de uma situação indesejável, como que emitindo sofrimento, fome, doença, medo, desconforto, estado emocional. Consequentemente, os ruídos/gritos podem indicar distúrbio no seu bem-estar (WEARY et. al., 1999; MANTEUFFEL et al., 2004; SAMPAIO, 2004). Para uma criação ser eficiente, é necessário prover ao animal ambiente adequado, de forma que possa ser expresso o comportamento normal de sua espécie, ao lidar ou identificar seus problemas (FRASER \& BROOM, 1990).

\section{CONCLUSÕES}

Os resultados apontaram que as variáveis térmicas encontradas no alojamento para reprodutores suínos e estudadas neste trabalho estão fora dos padrões ideais, enquanto a concentração de amônia não ultrapassou os limites recomendados na literatura. Quanto aos valores de níveis de ruídos, embora se possa classificar a instalação como adequada para trabalhadores, os valores de pico encontrados podem ser indicativo de condições inadequadas ao bem-estar animal. Com relação à análise feita utilizando a lógica fuzzy, foi possível traçar um cenário de bem-estar para os animais, em função das variáveis estudadas.

\section{AGRADECIMENTOS}

À FAPESP e ao CNPq, pelo auxílio à pesquisa.

\section{REFERÊNCIAS}

AMENDOLA, M.; CASTANHO, M.J.; NÄÄS, I.A.; SOUZA, A.L. Análise matemática de condições de conforto térmico para avicultura usando a teoria dos conjuntos Fuzzy. Biomatemática, Campinas, v.14, n.1, p.87-92, 2004.

AMENDOLA, M.; MOLLO NETO, M.; CRUZ. V. Using fuzzy sets to analyze environmental condition in order to improve animal productivity. Biomatemática, Campinas, v.15, n.1, p. 29-40, 2005.

AMENDOLA, M.; SOUZA, A. L.; BARROS, L. C. Manual do uso da teoria dos conjuntos fuzzy no Matlab r 6.5. Manual apresentado no Ciclo de Palestras/2004, realizado na FEAGRI/UNICAMP. Disponível em: <http://www.ime.unicamp.br/ laeciocb/manual_fuzzy_matlab.pdf >. Acesso em: 10 set. 2007.

BENEDI, J.M.H. El ambiente de los alojamientos Ganaderos. Madrid: Servicio de Extensión Agrária. 1986. 28 p.

BRUCE, J.M. Ventilation and temperature control criteria for pigs. In: CLARK, J.A. Environmental aspect of housing for animal production. London: Butterworths, 1981. p.197-216.

CIERESZKO, A.; OTTOBRE, J.S.; GLOGOWSKI, J. Effects of season and breed on sperm acrosin activity and semen quality of boars. Animal Reproduction Science, Wageningen, v.64, n.1, p.89-96, 2000 .

CIGR. COMMISSION INTERNATIONALE DU GÉNIE RURAL. Aerial environment in animal housing: concentrations in and emissions from farm buildings. Dublin: CIGR, 1994. 116 p.

Eng. Agríc., Jaboticabal, v.30, n.1, p.1-13, jan./fev. 2010 
CORCUERA, B.D.; HERNÁNDEZ-GIL, R.; ROMERO, C.A.; RILLO, SM. Relationship of environment temperature and boar facilities with seminal quality. Livestock Production Science, Amsterdam, v.74, n.1, p.55-62, 2002.

EASTMAN, J.R. IDRISI for Windows version 2 tutorial exercises. Worcester: Clark University, 1997.

FOOTE, R.H. Factors influencing the quantity and quality of semen harvested from bulls, rams, boars and stallions. Journal of Animal Science, Savoy, v.47, n.2, p.1-11, 1978.

FRASER, A.F.; BROOM, D.M. Farm animal behaviour and welfare. London: Baillière Tindall, 1990. $437 \mathrm{p}$.

HANSEN, D.G. Manejo del verraco destinado a la inseminación artificial: factores que afectan la fertilidad. In: SEMINÁRIO INTERNACIONAL DE SUINOCULTURA, 4., 1999, São Paulo. Anais... Concórdia: EMBRAPA Suínos e Aves, 1999. p.77.

HARDOIN, P.C. Qualidade do ar. Sistemas de ventilação natural e artificial na exploração avícola. In: SIMPÓSIO INTERNACIONAL SOBRE AMBIÊNCIA E INSTALAÇÃO NA AVICULTURA INDUSTRIAL, 1995, Campinas. Anais... Campinas: FACTA, 1995. p.89-98.

HENNESSY, D.P.; WILLIAMSON, P. The effects of stress and of ACTH administration in hormone profiles, oestrus and ovulation in pigs. Theriogenology, Standford, v.20, n.1, p.13-26, 1983.

HUANG, S.Y.; KUO,Y. H.; LEE, Y.P.; TSOU, H.L.; LIN, E.C.; LEE, W.C. Association of heat shock protein 70 with semen quality in boar. Animal Reproduction Science, Wagening, v.63, n.3, p.231-240, 2000.

JAINUDEEN, M.R.; HAFEZ, E.S.E. Distúrbios reprodutivos nos machos. In: HAFEZ, E.S.E. Reprodução animal. 6.ed. São Paulo: Editora Manole, 1995. p.291-301.

JENSEN, A.O. Changing the environment in swine buildings using sulfuric acid. Transaction of the $A S A E$, St. Joseph, v.45, n.1, p.223-227, 2002.

KASANEN, S.; ALGERS, B. A note on the effects of additional sow gruntings on suckling behaviour in piglets. Applied Animal Behaviour Science, Netherlands, v.75, n.2, p.93-101, 2002.

KLIR, G. J.; YUAN, B. Fuzzy sets and fuzzy logic. New Jersey: Prentice Hall PTR, 1995. 574 p.

KRISTENSEN, K.H.; WATHES, C.M. Amônia e bem-estar das aves: uma síntese. Clipping Merial de Avicultura, Campinas, v.2, n.6, p.27-32, 2001.

KUNAVONGKRIT, A.; PRATEEP, P. Influence of ambient temperature on reproductive efficiency in pigs: boar semen quality. Pig Journal, Escócia, v.35, n.2, p.43-47, 1995.

LARSSON, K.; EINARSSON, S. Seminal changes in boars after heat stress. Acta Veterinaria Scandinavia, Uppsala, v.25, n.1, p.57-66, 1984.

MACHADO FILHO, L.C.P. Aspectos do comportamento de suínos. In: ENCONTRO ANUAL DE ETOLOGIA, 6., 1998, Florianópolis. Anais ... Florianópolis: Imprensa Universitária da UFSC, 1988. p.88-105.

MALMGREN, L. Experimentally induced testicular alteration in boars. 1988. $20 \mathrm{f}$. Tese (Doutorado) - Swedish University of Agricultural Science, Uppsala, 1988.

MANTEUFFEL, G.; PUPPE, B.; SCHÖN, P.C. Vocalization of farm animals as a measure of welfare. Applied Animal Behaviour Science, London, v.88, n.1-2, p.163-182, 2004.

MARCHANT, J. N.; WHITTAKER, X.; BROOM, D.M. Vocalisations of the adult female domestic pig during a standard human approach test and their relationships with behavioural and heart rate measures. Applied Animal Behaviour Science, London, v. 72, n. 1, p. 23-39, 2001. 
MARTRENCHAR, A.; HUONNIC, D.; COTTE, J.P.; BOILLETOT, E.; MORISSE, J.P. Influence of stocking density, artificial dusk and group size on the perching behaviour of broilers. British Poultry Science, Abington, v.41, n.2, p.125-130, 2000.

MARX, G.; HORN, T.; THIELEBEIN, J.; KNUBEL, B.; BORELL, E. VON. Analysis of painrelated vocalization in young pigs. Journal of Sound and Vibration, Amsterdam, v.266, n.3, p.687698, 2003.

MCNITT, J.I.; TANNER, C.B.; FIRST, N.L. Thermoregulation in the scrotal system of the boar. I. Temperature distribution. Journal of Animal Science, Philadelphia, v.34, n.1, p.112-116, 1972.

MIES FILHO A. Fisiologia do aparelho genital masculino: função espermatogênica e função endócrina do testículo. In: MIES FILHO, A. Reprodução dos animais e inseminação artificial. 3.ed. Porto Alegre: Sulina, 1975, p.99-133.

MINITAB ${ }^{\circledR}$. Minitab Statistical Software for Windows. São Paulo: Award Brasil. 2004. Disponível em: <http://www.minitab.com/products/minitab>. Acesso em: 10 fev. 2004.

MOURA, D. J.; SILVA, W.T.; NÄÄS, I.A.; TOLON, Y.B.; LIMA, K.A.O.; VALE, M.M. Real time computer stress monitoring of piglets using vocalization analysis. Computers and Electronics in Agriculture, Leuven, v.64, n.1, p.11-18, 2008.

NÄÄS, I.A. Novas perspectivas da ambiência em relação ao bem-estar dos suínos e do trabalhador 19-6-2007. Disponível em: <http://www.porkworld.com.br/index.php?documento=111>. Acesso em: 10 ago. 2007.

NORMA REGULAMENTADORA. NR - 15: Atividades e operações insalubres. Disponível em: <http://www.mte.gov.br/temas/segsau/legislacao/normas/conteudo/nr15>. Acesso em: 10 jan. 2007.

OLIVEIRA, H.L.; AMENDOLA, M.; NÄÄS, I.A. Estimativa das condições de conforto térmico para avicultura de postura usando a teoria dos conjuntos fuzzy. Engenharia Agrícola, Jaboticabal, v.25, n.2, p.300-307, 2005.

OWADA, A.N.; NÄÄS, I.A.; MOURA, D.J.; BARACHO, M.S. Estimativa de bem-estar de frango de corte em função da concentração de amônia e grau de luminosidade no galpão de produção. Engenharia Agrícola, Jaboticabal, v.27, n.3. p.611-618, 2007.

PANDORFI, H. Comportamento bioclimático de matrizes suínas em gestação e o uso de sistemas inteligentes na caracterização do ambiente produtivo: suinocultura de precisão, 2005. 136f. Tese (Doutorado em Física do Ambiente Agrícola) - Escola Superior de Agricultura "Luiz de Queiroz", Universidade de São Paulo, Piracicaba, 2005.

PERDOMO, C.C.; LIMA, G.J.M.M.; NONES, K. Produção de suínos e meio ambiente. In: SEMINÁRIO NACIONAL DE DESENVOLVIMENTO DA SUINOCULTURA, 9., Gramado. Disponível em: <http://www.cnpsa.embrapa.br/>. Acesso em: 25 maio 2006.

RELATÓRIO. Relatório da Comissão ao Conselho e ao Parlamento Europeu sobre a Experiência Adquirida pelos Estados-Membros desde a entrada em vigor da Directiva 95/98/CE do Conselho que altera a Directiva 95/98/CEE. Conselho relativo às normas mínimas de proteção de suínos. 2001. Disponível em: <http://europa.eu.int/comm/food/>. Acesso em:10 set. 2006.

ROBERTSON, J.F. Dust and ammonia in pig buildings. Farm Building Progress, Aberdeen, v.110, n.1, p.19-24, 1990.

ROZEBOOM, K.; SEE, T.; FLOWERS, B. Coping with seasonal infertility in the herd: part I. 2000. Disponível em: <http://mark.asci.ncsu.edu/Swine_News/2000/sn_v2303.htm>. Acesso em: 4 jun. 2008.

SAMPAIO, C.A.P. Caracterização dos sistemas térmicos e acústicos em sistemas de produção de suínos nas fases de creche e terminação. 2004. 121f. Tese (Doutorado em Construções Rurais e 
Ambiência) - Universidade Estadual de Campinas, Faculdade de Engenharia Agrícola, Campinas, 2004.

SAMPAIO, C.A.P.; NÄÄS, I.A.; NADER, A. Gases e ruídos em edificações para suínos: aplicação das normas NR-15, CIGR e ACGIH. Engenharia Agrícola, Jaboticabal, v.25, n.1, p.10-18. 2005.

SAMPAIO, C.A.P.; NÄÄS, I.A.; SALGADO, D.D.; BARACHO, M.S. Avaliação de poluentes aéreos em instalações de creche e terminação de suínos. Ciência Rural, Santa Maria, v.37, n.2, p.488-494, 2007.

SCHNEIDER, L.G. Infertilidade estacional na produção de suínos. 2007. Disponível em: <www.Acsurs.Com.Br/Artigo\%20infertilidade\%20estacional>. Acesso em: 20 dez. 2007.

SEREN, E.; MATTIOLI, M.; RENSIS, F. Effect of high temperatures on LH and cortisol secretion in ovariectomized sows. In: INTERNATIONAL CONGRESS ON ANIMAL REPRODUCTION, 11., 1988, Dublin. Proceedings ... Dublin: ICAR, 1988. p.417.

SURIYASOMBOON, A.; LUNDEHEIM, N.; KUNAVONGKRIT, A.; EINARSSON, S. Effect of temperature and humidity on reproductive performance of crossbred sows in Thailand.

Theriogenology, New York, v.65, n.3, p.606-628, 2006.

SURIYASOMBOON, A.; LUNDEHEIM, N.; KUNAVONGKRIT, A.; EINARSSON, S. Effect of temperature and humidity on sperm morphology in duroc boars under different housing systems in Thailand. Journal of Veterinary Medicine Science, Tóquio, v.67, n.8, p.777-785, 2005.

WEARY, D.M.; APPLEBY, M.C.; FRASER, D. Responses of piglets to early separation from the sow. Applied Animal Behaviour Science, Londres, v.63, n.4, p.289-300, 1999.

WEBER, L.; KLEIN, P.A.T. Aplicações de lógica fuzzy em software e hardware. Canoas: Editora ULBRA, 2003. 112 p.

WETTEMAN, R.P.; BAZER, F.W. Influence of environmental temperature on prolificacy of pig. Journal of Reproduction and Fertility, Cambridge, v.33, n.1, p.199-208, 1985.

ZADEH, L.A. Fuzzy sets. Information and Control, Inglaterra, v.18, n.3, p.338-353, 1965. 